\title{
Performance Analysis of Multi-hop Technique on Two-tier Network Clustering in Wireless Sensor Network
}

\author{
Wan Isni Sofiah Wan Din ${ }^{1}$, Asyran Zarizi Abdullah ${ }^{2}$, RD Rohmat Saedudin ${ }^{3}$ \\ ${ }^{1}$ Faculty of Computing, Universiti Malaysia Pahang, Malaysia, sofiah@ump.edu.my \\ ${ }^{2}$ Politeknik Muadzam Shah, Department of Information Technology \& Communication, Malaysia, \\ asyran.abdullah@gmail.com \\ ${ }^{3}$ Telkom University, School of Industrial Engineering, Bandung, Indonesia, roja2128@ gmail.com
}

\begin{abstract}
Wireless Sensor Network (WSN) is a distributed connection that consists of many wireless sensor devices. It is used to get information from surrounding activities or environment and to send the details to the user for future computation. Due to its advantages, the WSN has been widely used to help people to collect, monitor and analyse the data. However, the biggest limitation of WSN is limited energy consumption which can affect the network performance. In order to reduce the energy usage, the process of selecting a path to the destination is very important. The current implementation uses the single-hop technique to send and receive data from source to destination and vice versa. The investigation shows that, the sensor nodes lifetime can be increased if some changes are made to the data transmission method. Therefore, the goal of this research is to increase energy efficiency using multi-hop technique as the primary transmission to make the network able to operate in a long period. An enhancement based on the Multi-tier Algorithm Protocol (MAP) has been produced. The enhancement technique is implemented to avoid direct communication between sensor nodes and cluster head, which is believed to be the main reason that caused inefficient use of energy (wastage). The proposed approach will give some advantages to data transmission, especially to the nodes that are located far from the cluster head. Subsequently, the experiment has been conducted to investigate the current implementation and this information has been used to make some improvements, before the performance of both techniques will be evaluated. The MATLAB is used to simulate and generate a graph. The results show that the enhancement algorithms will save energy up to $30 \%$ and is able to prolong the WSN lifetime with an advantage of 4462 cycles (to become 14283) compared to the original MAP which recorded only 9824 cycles.
\end{abstract}

Key words : Clustering, Energy, Multi-Hop, Wireless Sensor Network.

\section{INTRODUCTION}

In today's Internet of Things, Wireless Sensor Network have been used widely all around the world. The capability of its functions allows many applications to be implemented such as collecting the data in open area network, object tracking, monitoring for military, and surveillance [1], [2]. This wireless technology drastically changes the limitation on wired technology in most of the critical applications. By using wired technology, less application can be used as it only operates with a fixed connection and it has limited coverage range [3]. Additionally, this technology required high cost for the installation and maintenance compared to the wireless communication. The implementation of WSN will overcome the issues with wired connection and make the network more scalable and flexible to enable the important potential role in Internet of Things (IoTs) [4] [5].

The family of IEEE 802 standard is used to allow the communication in Wireless Sensor Network [6]. In WSN communication, single-hop transmission normally was used to transmit and received the data. Even though with existence of cluster head, the data transmission implementation still same except the cluster head will forward the data to the base station for the user use. Besides of single hop, multi hop also is important in clustering. Multi-hop has proved in increasing the WSN performance and can optimized the lifetime of WSN [7], [8]. Thus, there is an issue in single-hop communication which is energy conservation. Research show that, the direct communication between the sensor node and cluster head in clustering will imposed high energy usage. Plus, if the location of sensor node is far from the cluster head, it will deplete the energy fast than the sensor node that near to the cluster head [9]. Without proper design of the sensor node in clustering especially during data transmission, it will affect the whole process in WSN as the energy of the sensor will deplete fast. Due to that, solutions are needed to meet the requirements of the fast packet delivery and low delay transmission to save more energy and increase the lifetime of WSN. Hence, an implementation of multi-hop technique will solve the issues. According to [10], single-hop transmission 
consumes more energy than multi-hop. The use of multi-hop will minimize the energy usage especially in long distance connection and this will prolong the lifetime of WSN [11]. In this paper, we will evaluate both performances of single-hop and multi-hop techniques in two-tier network clustering. Both techniques will measure the energy consumption and network lifetime by several defined variable parameters. The remainder of this paper is structured as follows: the explanation of current implementation and the theory of the enhancement method will be discussed in section 2, the performance evaluation and simulation results where the comparison of both techniques has been discovered in section 3 , and section 4 contains the conclusion of the results and findings of the technique proposed.

\section{RELATED WORKS}

The large scale of network needs a multiple tier to perform a connection of multiple large nodes to the base station. The more nodes operating in a network, the more tiers are needed. It is used to prevent wasted energy from the direct connection activity. In this situation, it will be divided into two tiers of network which are tier 1 and tier 2. Each tier contains nodes with different tasks, with the same aim which is to send and receive data to and from the base station. The base station acts as the main network transmission component to the Internet located within 50 metres radius which is the centre of the network. The area of $100 \times 100$ metres will operate 100 randomly distributed static nodes. The area of circle formula $\left(\mathrm{A}=\pi \times \mathrm{r}^{2}\right)$ has been used to get the correct number of nodes distribution in both tiers. The amount of sensor nodes in the second layer needs to be tripled than the ones in the first layer which contains 25 nodes and the rest are located in tier 2 . The calculation details are shown in Figure. 1:

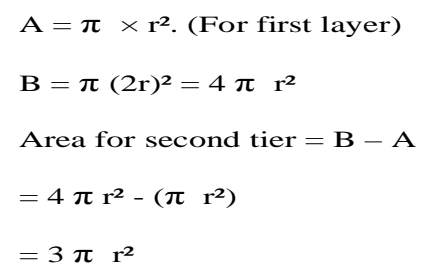

Figure 1: Calculation for nodes distribution in tier 1 and tier 2

The current implementation uses Multi-Tier Algorithm Protocol (MAP) [12] to select a cluster head that is able to work in WSN multiple tiers, using Fuzzy Logic. Based on the investigations [13], on the MAP transmission between nodes using single-hop, the lifetime of the sensor node can be increased if some changes are made to the data transmission. MAP uses 3 types of nodes: blue node represents normal sensor node, purple is secondary cluster head ( $\mathrm{SCH})$, and red is primary cluster head $(\mathrm{PCH})$. The centre of the cluster has a red rectangle as shown in Figure 4 which represents the base station. The PCH node has been selected by using K-Optimal formula. It is responsible for cluster formation and data forwarding to the base station. There are different amounts of PCH in both tiers. Tier 1 has 7 nodes and tier 2 has 12 nodes. Figure 2 shows the implementation of K-Optimal to distribute nodes of PCH.

kopt_tier1 $=$

round((sqrt(noTier1)/sqrt(2*pi) $\left.) * \operatorname{sqrt}(\operatorname{Efs} / E m p) *\left(M /\left(d 2 b s^{\wedge} 2\right)\right)\right)$;

Figure 2: K-Optimal formula for primary node placement

The noTier1 is the number of nodes in tier 1 which is 25 nodes, and 75 nodes for tier 2. The Efs is the amplification of energy when value $d$ is less than $d 0$. Emp is the amplification energy when $d$ is greater than $d 0$, and Etx is the transmitter energy per node. The distance to the base station (d2bs) is 50 metres. K-Optimal will distribute the $\mathrm{PCH}$ nodes in tier 1 and tier 2 as shown in Figure 3.
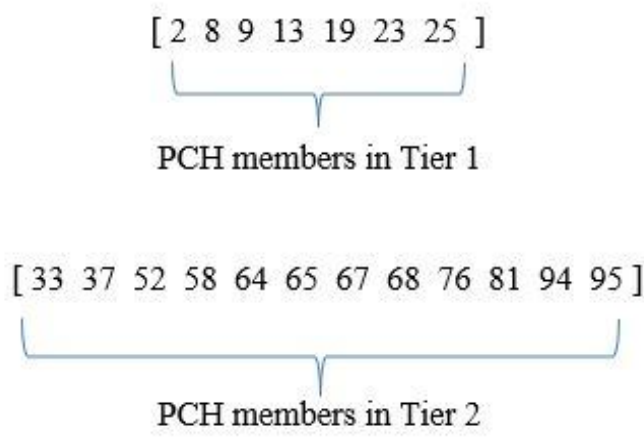

Figure 3: $\mathrm{PCH}$ member in tier 1 and tier 2

The SCH node is used to collect and compile the data before sending them to the PCH [14]. SCH will be randomly selected from the cluster groups. Each of the clusters should have 1 $\mathrm{PCH}$ and $1 \mathrm{SCH}$ to perform the network operation. Figure 4 shows the random distribution of 100 nodes allocated in tier 1 and tier 2. The connection of nodes in the network has been done by using two techniques which are single-hop and multi-hop. The previous researcher used single-hop to operate in MAP which produced positive results in terms of energy consumption compared to LEACH technique. Based on the previous experiment, LEACH recorded the last iteration of node at 4999, while MAP at 9824 [12]. 


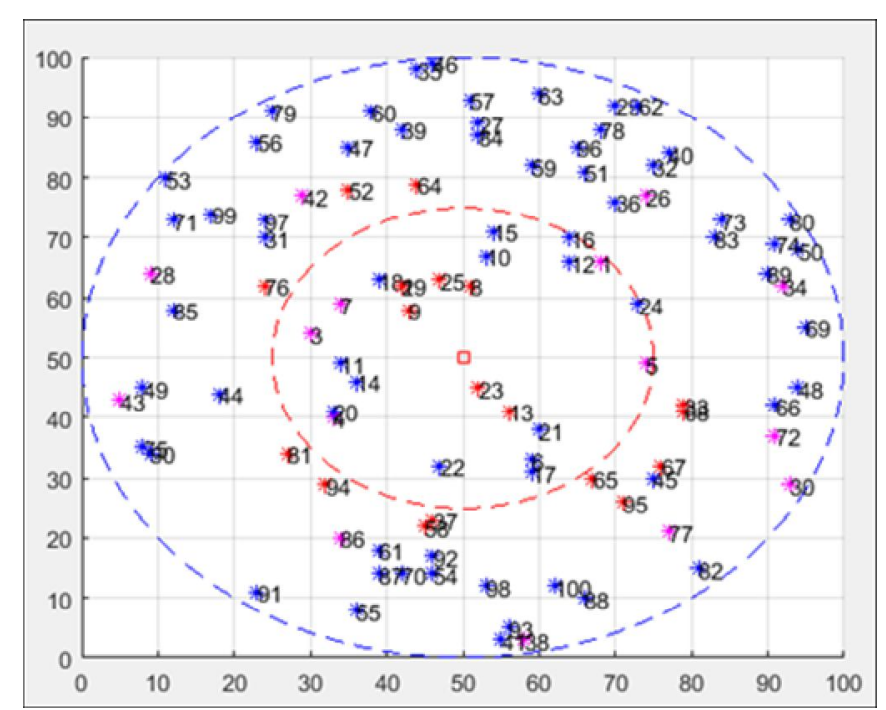

Figure 4: Node distribution in two-tier network clustering

Although the MAP needs a lot of changes to achieve their goals, the transmission method still uses the same technique which is single-hop. This can be improved to obtain better results. The single-hop transmission will connect from nodes to $\mathrm{SCH}, \mathrm{SCH}$ to $\mathrm{PCH}$ and $\mathrm{PCH}$ to the base station. This approach will waste energy when the nodes are located far from the destination [15] and need to carry multiple large data (10000 bits) to the SCH. This will affect the energy consumption with fast reduction of the nodes, making the nodes die when the energy is recorded below the threshold.

\section{MULTI-HOP TECHNIQUE}

The multi-hop transmission will operate in the same cluster group, and it will bring some good changes in terms of energy consumption. As shown in Figure 5(b), each of the nodes will send the data to the nearest node before sending to the $\mathrm{SCH}$, which is different with single-hop technique as shown in Figure 5(a), where all nodes will send the data directly to the $\mathrm{SCH}$. The implementation of both techniques has their strengths and weaknesses. The single-hop technique will send and receive data in one-to-one transmission. There will be no need to load more data from other nodes and will ensure the nodes have more space and time to run their tasks. Besides, the multi-hop approach will carry the data of other members to the destination which will burden and affect the energy of the nodes. In fact, the experiment shows that the first node dies faster in multi-hop compared to single-hop.

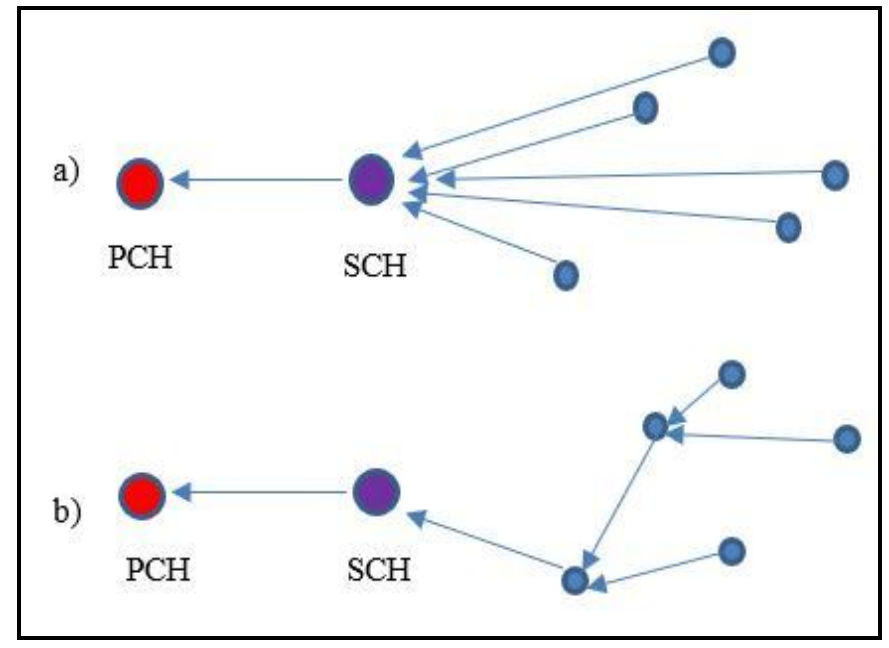

Figure 5: (a) Single-hop and (b) Multi-hop transmission

However, to prolong the lifetime of a network as stated in the objective, multi-hop technique is the best option [16]. It extends the last dead node of single-hop from 9824 to 14283 in 2000 bits data transmission, and also increases in all tested bits of data. The nodes located far from SCH will get an advantage. Using single-hop will take more energy to transmit the data to the destination, but the multi-hop nodes in the same spot will only send the data to the nearest node leading to an increased energy efficiency [17].

Multi-hop is implemented in WSN, where all of the sensor nodes are connected to each other as shown in Figure 6. The blue line represents the connection between nodes to nodes, whereas the connection of red line is between normal nodes and $\mathrm{SCH}$. The green line shows the connection between $\mathrm{SCH}$ to $\mathrm{PCH}$ and vice versa. This approach will help the nodes to save their energy and extend the network lifetime because there is no need to transmit the data in long transmission. But the nearest node has to be found in the same cluster group before sending the data.

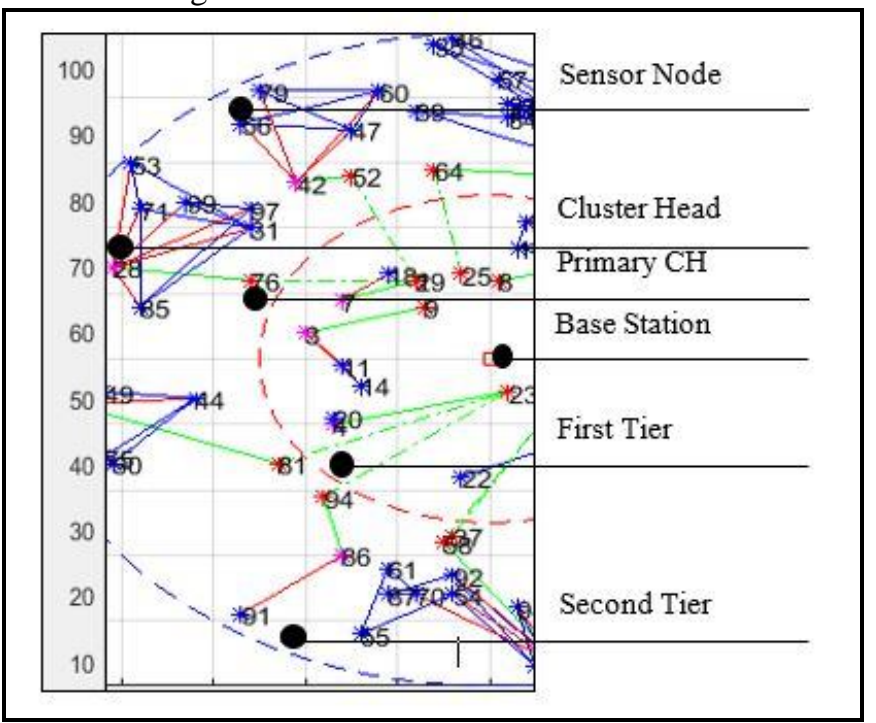

Figure 6: Multi-hop communication in MAP 
The radius is the length of circle from the centre to any node. It is used to find the nearest nodes between two types of connection in a cluster group, which can be either from $\mathrm{SCH}$ to other nodes and from nodes to nodes, as long as both connections will enable a multi-hop transmission. The coverage of radius is based on a distance formula between involved nodes as shown in Figure 7. It will calculate the distance between $\mathrm{SCH}$ and other nodes in a cluster group. For example, if the result of distance_node is 5 metres, then all nodes located in the distance of 5 metres will be able to make a connection.

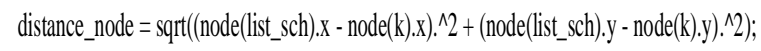

Figure 7: Distance calculation to find a neighbor

Furthermore, the radius determines the length of multi-hop coverage between any connection at the centre to any connected nodes in a group. A small radius will cover a small range of nodes, and a wider radius will cover a wider range of nodes as shown in Figure 8. Each of the cluster groups will have a distance measurement of their group. Based on the information gathered, the radius will find their path to enable a multi-hop connection. Figure 8(a) explains that in a configuration of 5 metres radius from the centre-point, it cannot make a connection to any node because the nearest node radius is more than 5 metres. Figure 8(b) shows the connection between centre-point to the nearest nodes in a radius of 8 metres.

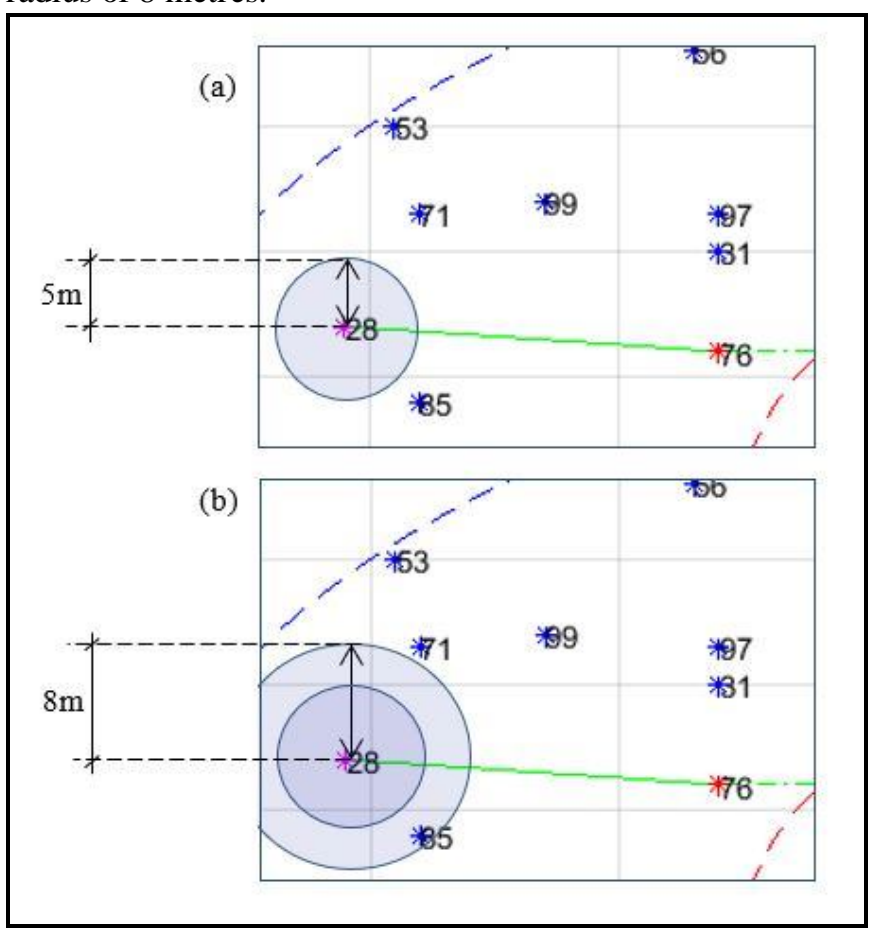

Figure 8: The use of radius in cluster group
To enable the multihop connection, the value of the radius must be increased to allow multiple connections from the centre-point to any range of radius. 15 metres of radius is used from the centre-point of node 71 as shown in Figure 8 and will create multiple paths to other connected nodes at coordinates $53,81,85$ and one direct connection to SCH which is node 28. All of the connected nodes will send and receive data to and from the center-point, before the node at the centre forwards all data to the connected SCH.

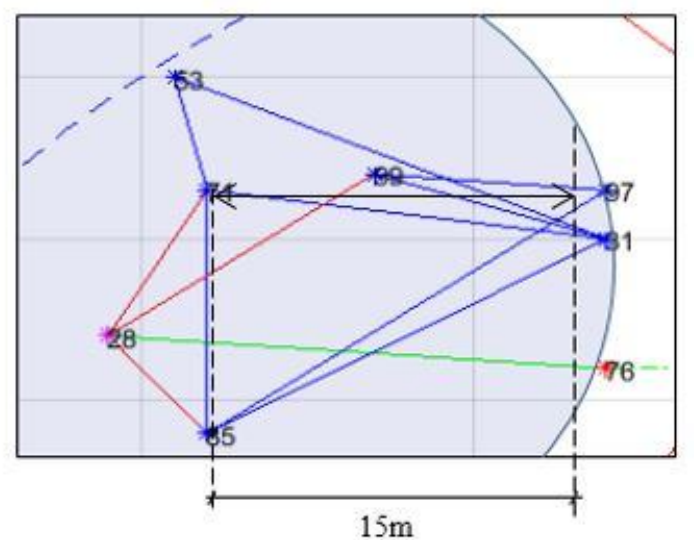

Figure 9: Multihop connection from center point of nodes

The algorithm of multihop connection shown in Figure 10 explains how the radius with a lower or equivalent value than distance_node will generate the multiple paths to other nodes in the radius range. If the value of the radius is greater than distance_node, the path will not be generated. This means the value is out of the cluster group range.

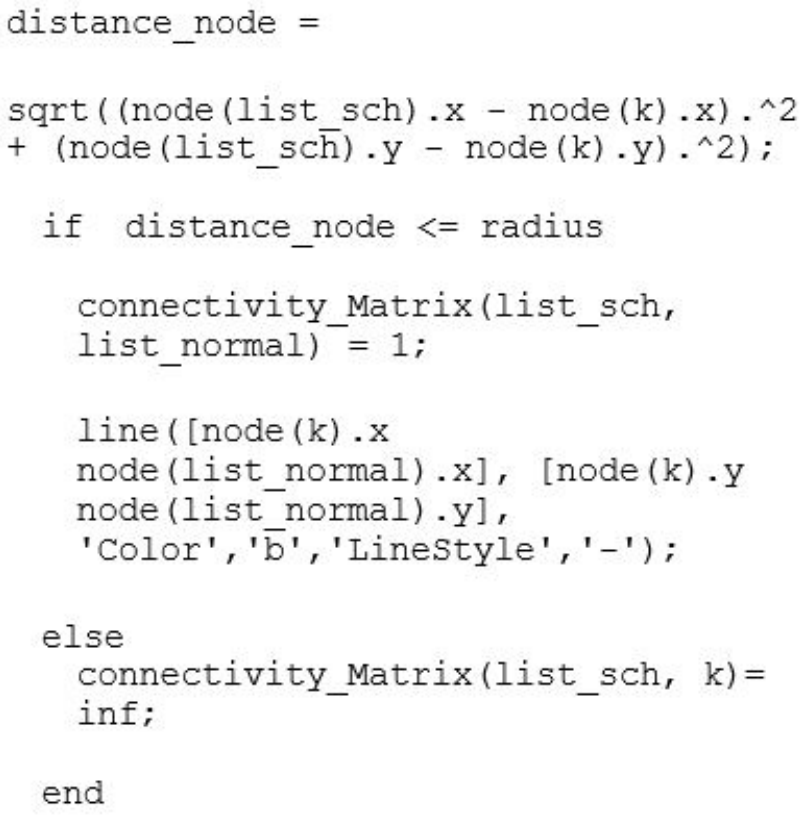

Figure 10: Implementation of multi-hop connection 


\subsection{Data Transmission Algorithm}

The operation of the current implementation will send the data directly from the node to cluster head. The data will be collected, aggregated and compressed by the cluster head before transmitted to the base station. Figure 11 shows the algorithm of data transmission performed in tier 1 and tier 2 .

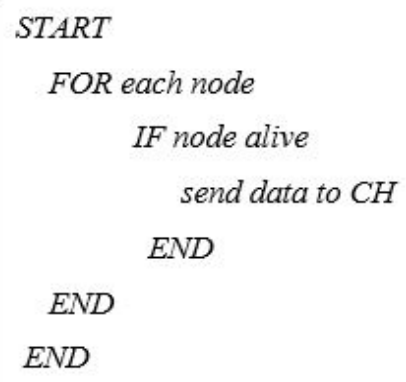

Figure 11: MAP Algorithm for sending a data to destination

Meanwhile, an enhancement of data transmission as shown in Figure 12 will improve the energy consumption where each node will find the nearest nodes to enable a multi-hop connection and prevent a long transmission.

START
For each node
IF node alive
find the nearest node from sender based on the radius.
build a multihop connection to $\mathrm{CH}$
send data to $\mathrm{CH}$ via multihop connection
END
END
END

Figure 12: An enhancement algorithm using multi-hop for sending a data to destination

\subsection{Energy Consumption Model}

This experiment will use 1 joule as the unit of energy for each node. Energy reductions occur based on node activity, where the node with zero joule is considered as a dead node [18]. The nodes using a lot of energy when carrying large data sizes are located far from the destination require more time and energy for data transmission. As a dead node cannot perform any task, the nearest node will take over the operation. The changing of path selection technique, from single-hop to multi-hop also requires a change in energy model. The single-hop energy is configured from all cluster group members to a single $\mathrm{SCH}$ and $\mathrm{PCH}$, while the multi-hop energy from cluster group nodes to nodes will find the shortest path to transmit the data before sending to the $\mathrm{PCH}$. The energy formula as shown in Figure 13 is used by computing the distance (d) between transmitter and receiver nodes. The distance will control and compensate for the loss of energy. If the distance (d) is less than the threshold value $(\mathrm{d} 0)$, the $\left(\mathrm{d}^{2}\right)$ model will be used. Otherwise, the $\left(d^{\wedge} 4\right)$ model is used.

$E_{T x}(k, d)=\left\{\begin{array}{l}k \times E_{\text {elec }}+k \times E_{f s} \times d^{2}, \text { if } d \leq d 0 \\ k \times E_{\text {elec }}+k \times E_{m p} \times d^{4}, \text { if } d<d 0\end{array}\right\}$ (1)

Figure 13: Transmission energy formula

The E_TX is the energy taken to transmit the packet. $E \_f s$ is the amount of energy consumption for the free space. E_elec is the energy dissipation of radio transceiver circuitry and $E_{-} m p$ is the energy needed for the transmit amplifier. The formula is based on the distance where (d) uses Pythagoras theorem to calculate the distance between nodes, while $d 0-\sqrt{\frac{E_{f s}}{E_{m p}}}$ is the threshold distance value. In MAP, all nodes are given a random and default energy. Based on the distance between each node, the energy will be calculated from the source to the destination node. In single-hop, the energy will be counted from one-to-one transmission, while in multi-hop, it will be counted from one-to-many destinations. Figure 14 shows the multi-hop energy configuration between nodes to nodes in MATLAB.

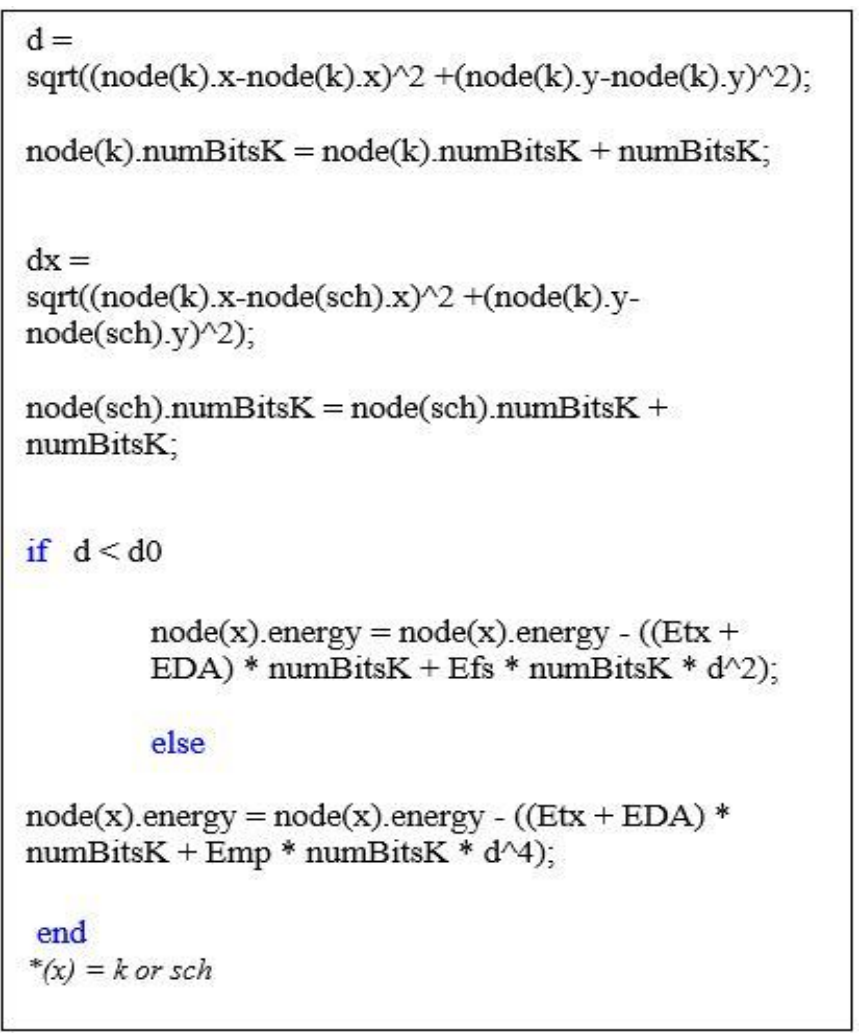

Figure 14: Distance calculation between connected nodes to nodes Each of the nodes needs to find the distance between the connected nodes. For example, node A will calculate the distance between $\mathrm{A}$ to $\mathrm{B}$ and $\mathrm{A}$ to $\mathrm{C}$. The distance is used to control the energy consumption in the network. If the distance is less than $\mathrm{d} 0$, it will use the low energy for data 
transmission. If the distance is above $\mathrm{d} 0$, then the high energy will be used.

\section{PERFORMANCE EVALUATION}

The implementation of multi-hop routing for path selection will reduce the energy usage and improves the network performance where the sensor node will send the data to the nearest node before the data is received by the base station. Logically, the previous implementation using single-hop will consume more energy and affect the network lifetime [19]. The comparison needs to be done to find the best approach for energy consumption in WSN environment. The goal of this project is to enhance the efficiency of energy usage to enable the sensor node to operate for a longer period. There are some techniques that can be used to achieve this goal. The way of selecting the best path in the network clustering [20] will reduce the load of node transaction between sensor nodes and cluster head. The experiment will use several parameters, where the number of nodes, maximum iteration, bits packet transaction and energy supply will repeatedly be changed to get different results. In this section, there are disclosures about the simulation results using several parameters. The one-to-one comparison between the current implementation and the new approach that used the same parameters and datasets has been discovered. The development process from nodes distribution, network tiers formation to running the code will be discussed. The results of both experiments will be analysed to determine the best solution to reduce energy consumption.

The MATLAB R2016a simulation tool is used to measure the performance of single-hop and multi-hop techniques. The simulation uses the parameters/datasets as shown in Table 1, where the length and width of the simulation area is 100 metres each. The number of nodes is 100 , with 1 joule of energy. The bits data transfer from minimum size of data starts from 2000 bits until it reaches 10000 bits, with an increment of 2000 bits for each cycle. This implementation will operate by 10000 and 15000 maximum iterations (only for 2000 bits data) where all parameters are tested in the three experiments. Graphs showing the results of sensor nodes maximum lifetime and average energy loss are produced to facilitate comparisons between both techniques.

Table 1: Parameter used in this simulation process

\begin{tabular}{|l|l|}
\hline Parameter & Value \\
\hline Network Simulator & Matlab R2016a \\
\hline Technique & Singlehop \& Multihop \\
\hline Simulation Area & $100 \times 100$ meter \\
\hline MAC Type & 802.11 IEEE \\
\hline No. of nodes & 100 nodes \\
\hline Maximum Iteration & 15000 \\
\hline Enegy & 1 joule \\
\hline Number of bits & $\begin{array}{l}2000, \quad 4000,6000,8000, \\
10000\end{array}$ \\
\hline
\end{tabular}

The performance of single-hop and multi-hop techniques in terms of energy consumption and maximum sensor nodes lifetime will be analysed. The goal of this experiment is to prove the usage of multi-hop technique will save more energy and is able to extend the network lifetime compared to single-hop technique. The graphs and results of the number of nodes are as shown in Figure. 15, 16 and Table 2.

The experiment has been done with 10000 iterations (2000 bits use 15000 iteration) with the same datasets and energy supply. In Figure 15 and 16, they show the differences between single-hop and multi-hop techniques with the focus on the first and last node dead. When more nodes survived the long iteration cycles, it proves that efficient use of energy has been successfully implemented. Overall, it can be seen that the first node of multi-hop will die earlier than single-hop, as shown in Figure 15(e). The first node found dead in multi-hop technique is at the 92nd iteration. Single-hop technique recorded its first dead node at iteration number 1462. The same pattern of information can be seen in (a), (b), (c), and (d) as shown in Table 2. The size of the bits will affect the lifetime of the nodes. The small size of the bits will undergo the maximum iteration as performed by 2000 bits, while the size of the large bits (10000 bits) will be burdened to transmit the data and caused it to die earlier compared to other bits.

There is a positive increase of multi-hop technique in terms of energy usage, where at 2000 bits it saved 30\% of energy compared to single-hop. At 4000 bits, it recorded $71.42 \%$ of the energy supplied, while single-hop recorded only $49.12 \%$. There is a difference of approximately $22.3 \%$ of energy consumption that is not fully utilised by single-hop. At 6000 bits of data, single-hop wasted around $14.87 \%$ of energy compared to multi-hop which registered an increase to 47.63\%. The 8000 and 10000 number of bits also shows an increment in multi-hop technique with $11.16 \%$ and $8.93 \%$ compared to the current implementation technique.

Table 2: The first and last node dead in a 2000 until 10000 bits of transfer

\begin{tabular}{|c|c|c|c|c|}
\hline \multirow{2}{*}{$\begin{array}{c}\text { No of } \\
\text { bits }\end{array}$} & \multicolumn{2}{|c|}{ Multi-hop } & \multicolumn{2}{c|}{ Single-hop } \\
\cline { 2 - 5 } $\mathbf{1}^{\text {st }}$ dead & $\begin{array}{c}\text { Last } \\
\text { dead }\end{array}$ & $\mathbf{1}^{\text {st }}$ dead & $\begin{array}{c}\text { Last } \\
\text { dead }\end{array}$ \\
\hline 2000 & 458 & 14283 & 7309 & 9824 \\
\hline 4000 & 229 & 7142 & 33655 & 4912 \\
\hline 6000 & 153 & 4763 & 2437 & 3276 \\
\hline 8000 & 115 & 3573 & 1828 & 2457 \\
\hline 10000 & 92 & 2859 & 1426 & 1966 \\
\hline
\end{tabular}



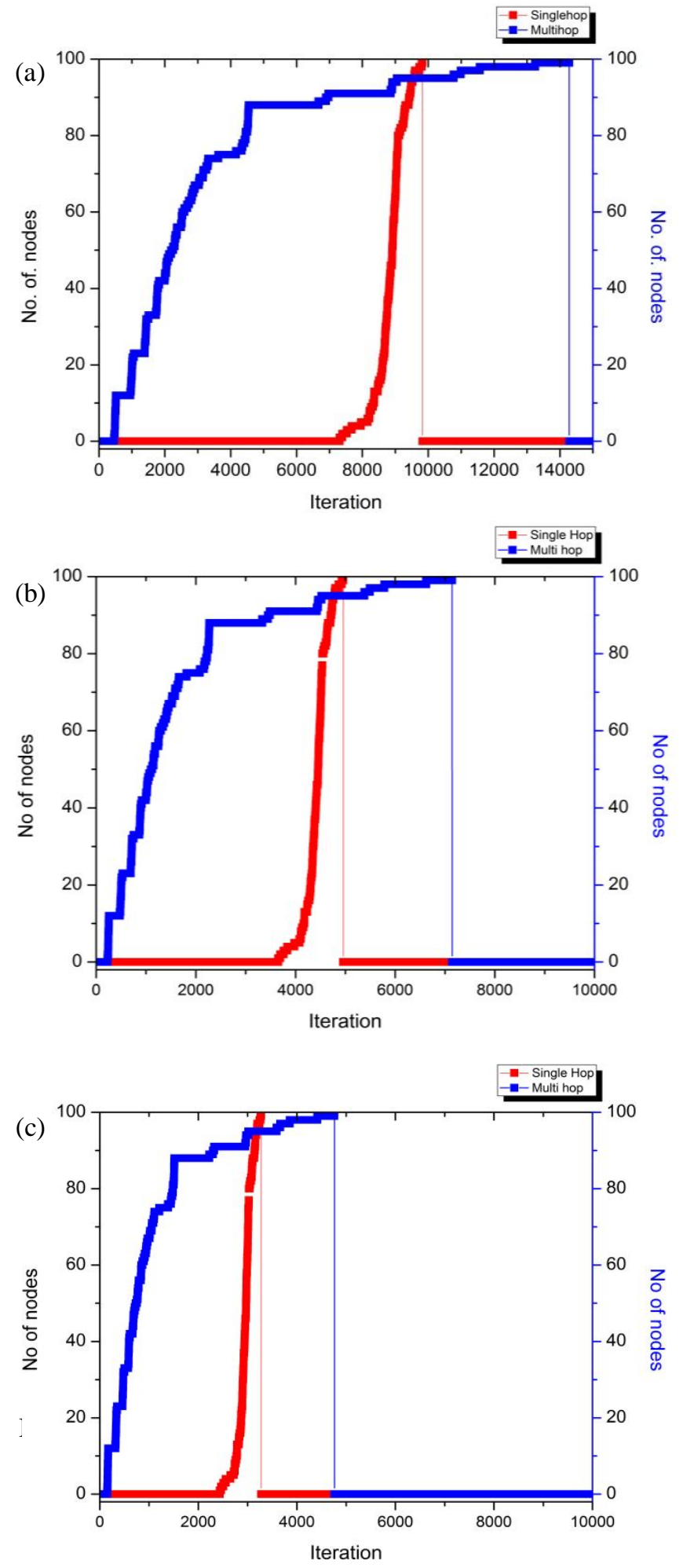

Figure 15: Number of dead nodes in single-hop and multi-hop for (a) 2000 bits

(b) 4000 bits and (c) 6000 bits.
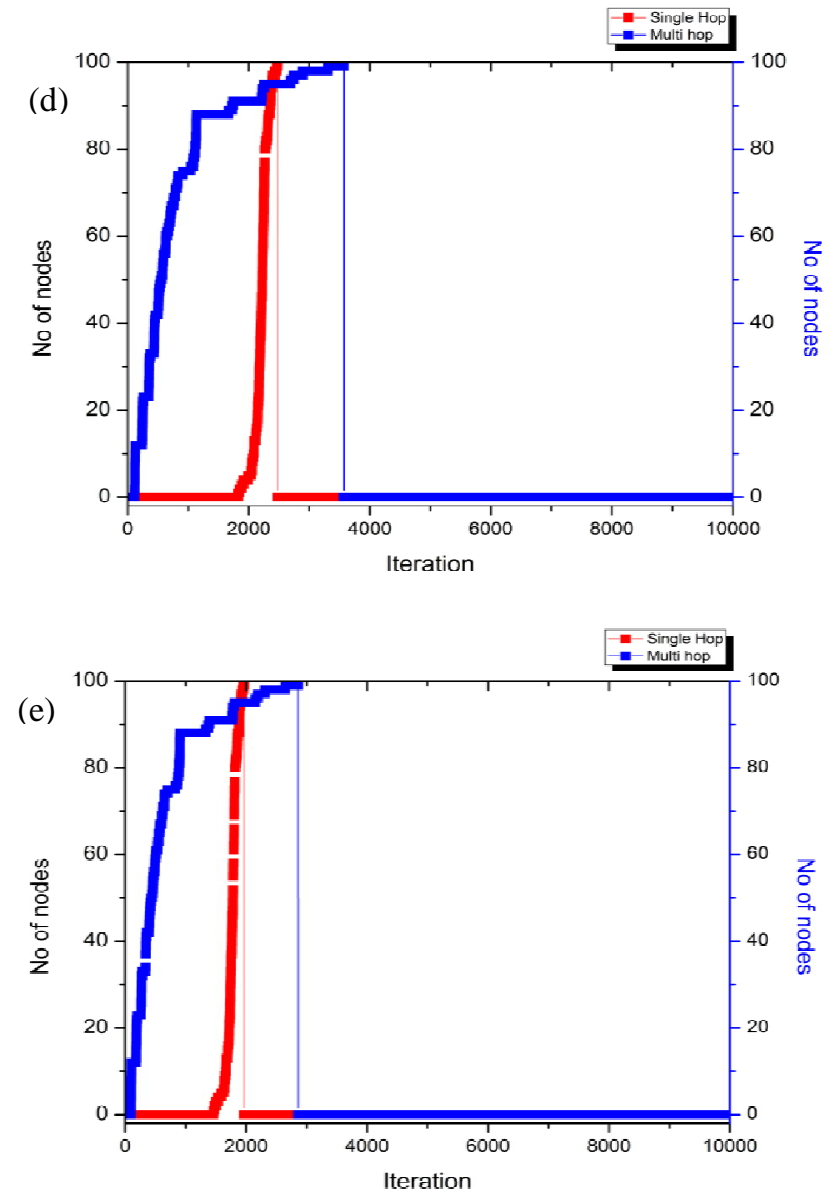

Figure 16: Number of dead node in single-hop and multi-hop for (d) 8000 bits, (e) 10000 bits

There is a big difference in both techniques. Single-hop will carry their own data to the destination $(\mathrm{SCH})$, but multi-hop will carry other the data of other nodes (based on the nearest neighbor using radius) to the destination. The more data is carried the more energy will be used [21]. Although the nodes in multi-hop technique are detected to die first than single-hop, the operation utilises energy efficiently and multi-hop technique is also able to extend the network lifetime [22]. It can be observed that the last dead node of multi-hop has a longer iteration cycle than single-hop as seen in Figure 17 where it shows the comparison of both techniques at each stage.

The performance using multi-hop technique is more energy-saving compared to single-hop. Although the first node dies faster with multi-hop compared to single-hop, multi-hop is the best technique to achieve maximum iteration at each cycle. The results of both implementations prove that multi-hop technique fully utilises the supplied energy efficiently and saves on average 38\% of energy compared with current implementation using single-hop technique when the same energy level is used. 


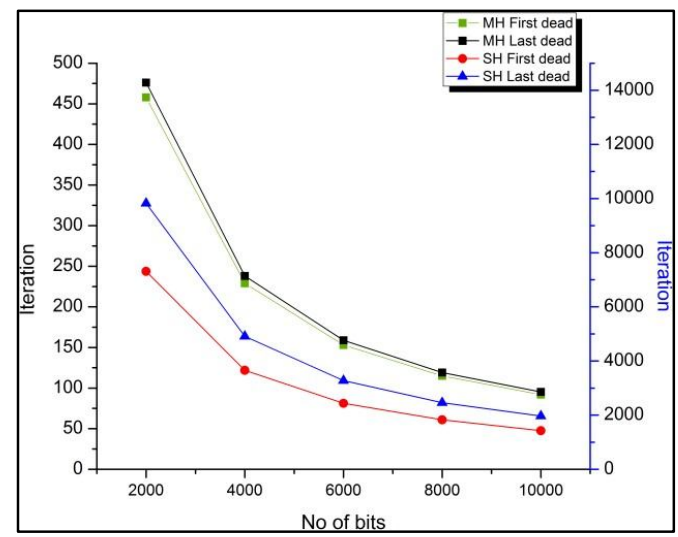

Figure 17: Number of node first and last dead in multi-hop (MH) and single-hop (SH) technique

The average energy loss for both techniques has been analysed. The results are as shown in Table 3 . The experiment uses the same bits of data where the maximum iteration is 15000 with 1 joule of energy to operate at different number of bits (2000 to 10000).

Table 3: The average energy loss in multi-hop and single-hop technique

\begin{tabular}{|c|c|c|}
\hline Bits & Single-hop & Multi-hop \\
\hline $\mathbf{1 0 , 0 0 0}$ & 0.11321 & 0.06234 \\
\hline $\mathbf{8 0 0 0}$ & 0.14182 & 0.07085 \\
\hline $\mathbf{6 0 0 0}$ & 0.17562 & 0.08374 \\
\hline $\mathbf{4 0 0 0}$ & 0.24738 & 0.10794 \\
\hline $\mathbf{2 0 0 0}$ & 0.42117 & 0.18312 \\
\hline
\end{tabular}

The multi-hop technique is able to fully utilise the energy given where it can perform until 14283 iterations, while single-hop records a maximum iteration of only 9824 . The new approach also shows the efficiency of energy usage as shown in Figure 18 where it only uses a total of 1.8703 joules energy for 10000 bits transmission and can operate until 2859 iterations (average is 0.06234 at each cycle). In the current implementation, it uses 3.2831 joules (average is 0.11321 at each cycle) for only 1966 iterations.

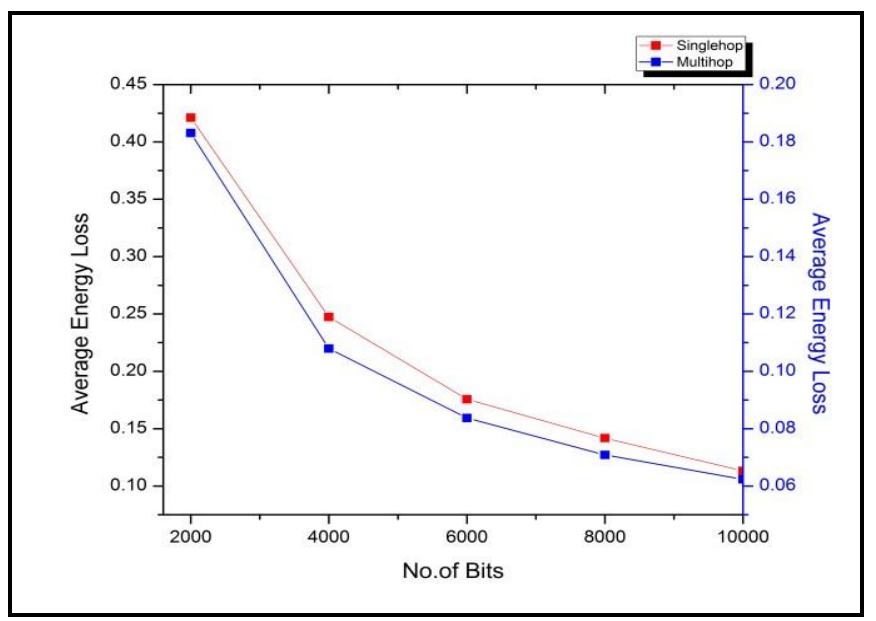

Figure 18: Average energy loss in new approach

The energy distribution is based on how many nodes remain.
The multi-hop technique causes nodes to die faster. When only several nodes are still active, the energy will be distributed to a small cluster group and saves more energy. However, the single-hop technique has to distribute the energy to many cluster groups. The data shows that at 2000 bits and 8600 iterations, the multi-hop recorded 91 dead nodes and only 9 were active. Single-hop reported that 18 nodes died. Therefore, the distribution of energy for 9 and 82 nodes will make a huge difference in terms of energy saving [23].

\section{CONCLUSION}

In this paper, the performance analysis of single-hop and multi-hop techniques on two-tier network clustering using various parameters has been done. Overall, the algorithm has successfully achieved the objective. Based on the results, it shows that the multi-hop performance is better than single-hop in terms of energy consumption and network lifetime. The technique will save $38 \%$ of energy and is able to operate in a longer period compared to single-hop. Furthermore, it can also extend the node lifetime [24]. The original MAP can only operate up to 9824 iterations, but the implementation of multi-hop technique can reach up to 14284 iterations (with the advantage of 4459 cycles) with $30 \%$ increment from the previous implementation. This paper can also be enhanced by implementing other multi-hop routing protocol with different parameters and metric units which can produce a more accurate result [25]. Plus, the coordination of the sensor nodes could be improved by using other approach such as K-Means and the energy consumption will be conserved during data transmission either in single-hop or multi-hop.

\section{ACKNOWLEDGEMENT}

The author would like to thank Melissa Chin for providing proof reading in this article. We would like to acknowledge and thank RACER/1/2019/ICT03/UMP//1: RDU-192622 in providing the financial support and facilities for this study. We are also grateful and thank the Faculty of Computer System \& Software Engineering UMP and Systems Network $\&$ Security Research Group (Sysnets) that has supported this research.

\section{REFERENCES}

1. L. Muduli, P. K. Jana, and D. P. Mishra (2018). Wireless sensor network based fire monitoring in underground coal mines: A fuzzy logic approach. Process Saf. Environ. Prot., vol. 113, 435-447.

2. M. Elhoseny, A. Tharwat, X. Yuan, and A. E. Hassanien (2018). Optimizing K-coverage of mobile WSNs. Expert Syst. Appl., vol. 92, 142-153. 
3. I. Del Castillo, F. Tobajas, R. Esper-Chaín, and V. De Armas (2016). Hardware platform for wide-area vehicular sensor networks with mobile nodes. Veh. Commun., vol. 3, 21-30.

4. Y. H. Lin, Z. T. Chou, C. W. Yu, and R. H. Jan, (2015). Optimal and Maximized Configurable Power Saving Protocols for Corona-Based Wireless Sensor Networks. IEEE Trans. Mob. Comput., vol. 14, no. 12, 2544-2559.

5. K. Okokpujie, M. Odusami, E. Noma-Osaghae, O. Modupe, S. John, and O. Oluwatosin (2018). A Smart Air Pollution Monitoring System. Int. J. Civ. Eng. Technol. (IJCIET, vol. 9, no. 9, 799-809.

6. S. G. Santhi and B. Divya (2015). Energy Consumption using IEEE802.15.4 Sensor Networks. Int. J. Comput. Appl., vol. 116, no. 9, 30-33.

7. P. Kuila and P. K. Jana (2012). Energy Efficient Load-Balanced Clustering Algorithm for Wireless Sensor Networks. Procedia Technol., vol. 6, 771-777.

8. J. A. Nayak (2017). Improving the Network Life Time of Wireless Sensor Network using EEEMR Protocol with Clustering Algorithm. Int. J. Electron. Electr. Comput. Syst. IJEECS, vol. 6, no. 6, 342-346.

9. P. Sharma and A. Gupta (2014). A Review on Multiple Single Hop Clustering Based Data Transmission in Wireless Sensor Network. vol. 3, no. 7.

10. K. Selvakumar and R. M. Arieth (2017). Contrast for QOS based clustered energy efficient protocol with PSO and multi-hop gateways in wireless sensor network.

11. A. A. Khamiss, C. Senchun, Z. Baihai, and L. I. Qiao (2014). Energy- Balanced Improved Leach Routing Protocol For Wireless Sensor Networks. 1-11.

12. W. I. S. W. Din, S. Yahya, M. N. Taib, A. I. M. Yassin, and R. Razali (2014). MAP: The new clustering algorithm based on multitier network topology to prolong the lifetime of wireless sensor network. Proc. IEEE 10th Int. Colloq. Signal Process. Its Appl. CSPA, 173-177.

13. E. K. Hamza (2018). Energy Consumption Analyzing in Single hop Transmission and Multi-hop Transmission for using Wireless Sensor Networks. Al-Khwarizmi Eng. Journal, vol. 14, no. March, 156-163.

14. R. Velmani and B. Kaarthick (2014). An Energy Efficient Data Gathering in Dense Mobile Wireless Sensor Networks. ISRN Sens. Networks, vol. 2014, 10.

15. S. Nithyakalyani (2013). Data Aggregation In Wireless Sensor Network Using Node Clustering Algorithms - A Comparative Study. IEEE Conf. Inf. Commun. Technology, 508-513.

16. J. Li and G. Alregib (2009). Network Lifetime Maximization for Estimation in Multihop Wireless Sensor Networks. IEEE Trans. SIGNAL Process., vol. 57, no. 7, 2456-2466.

17. S. L. P, M. G. Jibukumar, and V. S. Neenu (2018). Network Lifetime Enhancement of Multi-Hop Wireless Sensor Network by RF Energy Harvesting. Int. Conf. Inf. Netw, 738-743.

18. M. Esmaeeli and S. A. H. Ghahroudi (2016). Improving energy efficiency using a new game theory algorithm for wireless sensor networks. Int. J. Comput. Appl., vol. 136, no. $12,1-4$.

19. L. Lin, C. Yang, K. J. Wong, H. Yan, J. Shen, and S. J. Phee (2014). An energy efficient MAC protocol for multi-hop swallowable body sensor networks. Sensors (Switzerland), vol. 14, no. 10, 19457- 19476.

20. R. B. P. F. Dilip Kumar (2011). Multi-Hop Data Communication Algorithm for Clustered Wireless Sensor Networks. Int. J. Distrib. Sens. Networks.

21. C. Wu, T. Yoshinaga, X. Chen, L. Zhang, and Y. Ji (2018). Cluster-Based Content Distribution Integrating LTE and IEEE 802.11p with Fuzzy Logic and Q-Learning. IEEE Comput. Intell.Mag., vol.13, no.1, 41-50.

22. A. Saxena et al (2017). Review of clustering techniques and developments. Neurocomputing, vol. 267, 664-681.

23. A. S. Rostami, M. Badkoobe, F. Mohanna, H. Keshavarz, A. A. R. Hosseinabadi, and A. K. Sangaiah (2018). Survey on clustering in heterogeneous and homogeneous wireless sensor networks. J. Supercomput., vol. 74, no. 1, 277-323.

24. A. A. Allahham and M. A. Rahman (2018). A Smart Monitoring System for Campus Using Zigbee Wireless Sensor Networks. Int. J. Softw. Eng. Comput. Syst., vol. 4, no. 1, 1-14.

25. T.-T. Nguyen, J.-S. Pan, S.-C. Chu, T.-K. Dao, and V.-C. Do (2019). Improved Performance of Wireless Sensor Network Based on Fuzzy Logic for Clustering Scheme. Adv. Smart Veh. Technol. Transp. Commun. Appl. VTCA 2018. Smart Innov. Syst. Technol. Springer, vol. 128, 104-113. 